\title{
WLAN Location Determination in e-Home via Support Vector Classification
}

\author{
Chao-Lin $\mathrm{Wu}^{1}$, Li-Chen Fu ${ }^{1,2}$, Feng-Li Lian ${ }^{2}$ \\ Department of Computer Science \& Information Engineering ${ }^{1}$ \\ Department of Electrical Engineering ${ }^{2}$ \\ National Taiwan University, ROC
}

\begin{abstract}
Because of the advanced development in computer technology, home automation system could provide a variety of convenient and novel services to people. But only providing many kinds of services is not enough; instead, upgrading the quality of services is also a very important issue. One way to upgrade the service quality is to customize the service according to the inhabitant's personal situation, and the user location is the key information for the home automation system to customize the services. Another impact of the advanced computer technology is to make the personal digital device to commonly have the capability to communicate through the wireless networks, and the popularity of wireless networks in home has increased in recent years. As a result, home automation system can bring services to personal digital devices held by people through any wireless network, and customize the services according to the location of personal digital device in home. In this paper, we present a location determination system for the home automation system to provide location aware services. This location determination system uses support vector machine to classify the location of a wireless client from its signal strength measures, and we will describe its architecture and discuss its performance.
\end{abstract}

\section{Introduction and Motivation}

Beneficial from the advanced development in computer technology, the home control network can connect the conventional electronic appliances together and provide them the ability to communicate with the personal computer (PC). As for another important role in e-Home, the information appliance (IA), it has the ability to share information with others, and the latter gives the $P C$ the mechanism to communicate with IA. Since all the appliances can communicate among one another via the $\mathrm{PC}$, the personal computer-based environment is the best platform for system integration. Taking advantage of the powerful computation capability of PC, the home automation system, an integration of the current hardware and software technologies, can be developed easily so as to provide a variety of services to the inhabitants [1] - [3].

Clearly, only providing these integrated services is not enough, whereas upgrading the quality of services is also an important issue. One of the ways to improve the quality is to customize the service according to the personal situation. Among all the personal information, the user location is a very useful one for the home automation system to customize the service. Because people's activity is always limited to the living space, and the resource mostly used by people are located in the space close to the people. Therefore, the goal of this paper is to develop a location determination system for the home automation system so as to provide location aware service. The basic idea is to propose the mechanism to detect the location of the personal mobile device.

To provide the location aware services, many systems have been developed over the years. Some of them require special hardware to detect the location of the mobile devices, like infrared-based systems [4], and computer vision systems [5]. Some of them could work only outdoors, like GPS [6]. Without requiring extra hardware and for suitability in indoor environment, many systems based on radio frequency (RF) are developed in recent years [7] - [14]. The concept of the RF-based systems is to use the underlying wireless data network such as IEEE 802.11 (Wi-Fi), to estimate the location of the client, i.e., the personal mobile device.

The popularity of wireless networks in home has increased in recent years, and the advanced computer technology has made the personal digital device to commonly have the capability to communicate through the wireless network. Hence, it is suitable to use RF-based location determination system to estimate the location of the personal digital device in a home environment.

Among all these RF-based location determination systems, they generally fall into two categories [7]:

1. Centralized Tracking System:

A central infrastructure will collect the signal strength information from the mobile clients, then infer the location of these wireless clients.

2. Distributed Beacon System:

The clients will infer its location by its own, through the signal strength information collected from the wireless access point they see in the environment. 
The client of the system belonging to the second category will need both the capability to detect the signal strength and enough computation resources to infer its location. However, the personal digital device used in home may not be qualified for those conditions mentioned above. On the other hand, the performance might be degraded when personal digital device does these computations, which will not be the situation people prefer.

The most controversial issues of the system belonging to the first category are:

1. User Privacy:

Since the location inference is done by the centralized system, the system will keep all the information and always know the location of the clients.

2. Network Burden:

To infer the location of the client, the system will keep collecting the wireless signal strength from the clients all the time. The necessity to obtain these data and send them to the server for computing will increase the burden of the network traffic.

The first issue will not be a problem at home because people who can see all the information of the system will be the family member only, and people will not be afraid of being known where he is by other family members. As for the second issue, the number of the clients will be much lower than the one in the office building or in the campus, so the burden of the network traffic will also be much lighter.

The system proposed in this paper belongs to the first category. The home automation system will integrate all the home services and provide the system control webpage for the inhabitants to access through wireless network. The location determination system will infer the location of the client according to its wireless signal strength, and the home automation system will automatically provide the suitable service to the inhabitants according to the inferred location.

The rest of the paper is organized as follows. Section 2 presents the general architecture for location determination system. The detail of the experiment is described in Section 3 and the system performance is evaluated in Section 4. In Section 5, three applications through the cooperation of location determination system and home automation system are proposed. Conclusions are made and some future works are discussed in Section 6.

\section{System Architecture and Specification}

The architecture of the proposed location determination system can be divided into two components: wireless signal sensor and location inference server. There will be multiple sensors, but only one server is needed. Figure 1 shows the relationship between these two components.

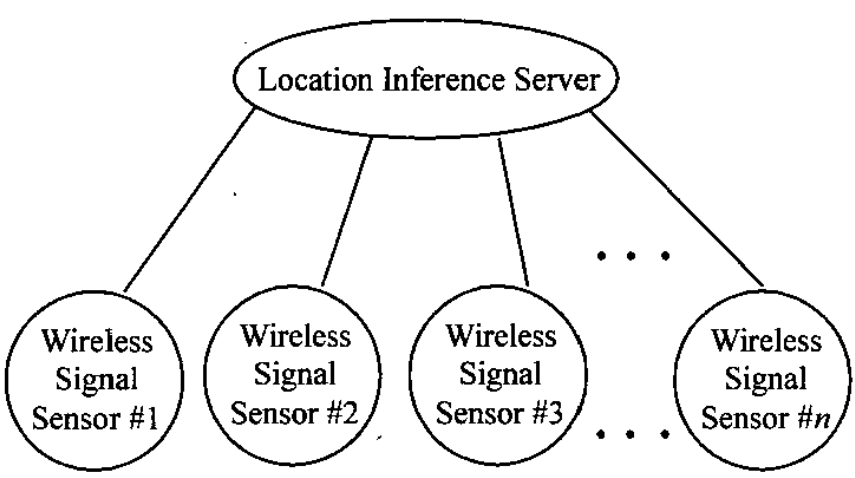

Figure 1. Relationship between Sensor and Server

\subsection{Wireless Signal Sensor}

Its task is to collect the wireless signal strength from wireless clients and then transmit data to location inference server. The working procedure of the wireless signal sensor is:

1. The operating system uses the wireless network card to collect the signal strength values from each wireless client in the environment.

2. Whenever the data is collected, the operating system will immediately send the collected data to the location inference server through the Ethernet card.

Among the information sent from wireless signal sensor to the location inference server, the crucial ones are:

1. Client Identity:

In the proposed system, multiple clients are detected by the sensors. Since the collected information is client dependent, the location inference server has to know which client the information belongs to. The Mac Address of the wireless network card used by the client is defined as the client identity.

2. Signal Strength:

This is the most important information because this information has strong dependence on the location of the wireless client.

3. Collecting Time:

The wireless client may change its location from time to time and hence will generate different signal strength information. If the information used by the location inference server is collected at some time instant other than the current one, the inferred location will be very likely wrong. Therefore, knowing the time of collecting the signal strength 
information is also very important.

4. Sensor Identity:

Without knowing by which sensor the information is collected, the set of the signal strength measures collected at some place may be the same as the one at other places. So it is also necessary to identify the sensors. For this purpose, one simple name is used for each sensor.

As for the client identity, although the Mac Address can be translated into some other simple name meaningful for people, this work has to be done in each sensor and this burden will be enormous if the number of sensors is large. It will be a great drawback when scalability becomes a key issue. Therefore, the translation work should be left to the location inference server. On the other hand, such name translation work will happen only when a new sensor is added, and hence the burden should be acceptable. Assigning each sensor a simple name meaningful to people not only relaxes the translation burden imposed on the location inference server but also allows people to more conveniently set up, monitor, and debug the system. It is worth mentioning in particular that the location inference server only cares the sensor from which the information is sent, not the underlying Ethernet card.

\subsection{Location Inference Server}

Its task is to infer the location of each wireless client according to the data collected by the sensors. Figure 2 shows the architecture of the location inference server.

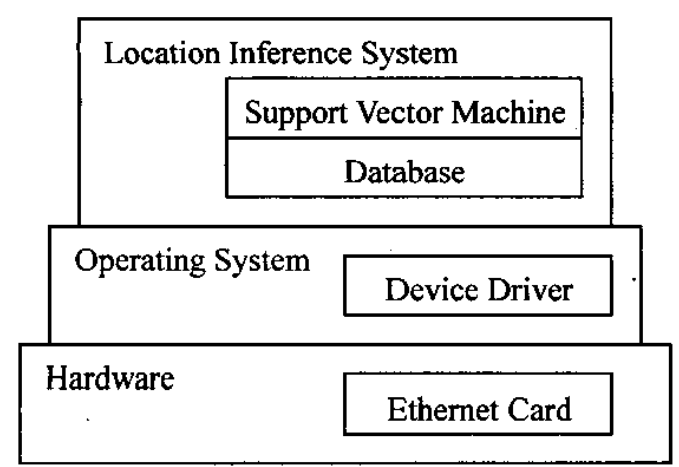

Figure 2. Location inference server architecture

The hardware layer is a simple PC equipped with the Ethernet card only. The operating system layer includes the operating system and the device driver whose task is for the operating system to interact with the Ethernet card. The location inference system has two important components:

1. Database:

Its task is to store and access the information sent from wireless signal sensor.

2. Support Vector Machine (SVM) :

SVM is a new and promising technique for data classification. It is a tool for statistic analysis and machine learning, and it performs very well in many classification applications. The LIBSVM (A Library for Support Vector Machines) [16] is chosen as the tool to infer the location of the wireless client.

The working procedure of the location inference server can be divided into two phases: off-line and on-line. Both of these two phases will be described in the following sections.

\subsubsection{Off-line Phase of the Location Inference Server}

This phase is also called the training phase according to the purpose of SVM. The off-line working procedure of the location inference server is described as follows:

1. The operating system receives the information from each sensor through the Ethernet network card.

2. The location inference system will parse the received information and store them into the database.

3. When storing the information into the database, the location inference system will also retrieve the relevant information as the training data for SVM to train the model for inference purpose. Because SVM requires that each data instance is represented as a vector of real numbers, the client identity will be converted into numeric data when being stored as the training data for SVM.

The other related details of the implementation will be described in the next section.

\subsubsection{On-line Phase of the Location Inference Server}

This phase is aiso called the testing phase according to the purpose of SVM. The on-line working procedure of the location inference server is:

1. The same as Step 1 in the off-line phase.

2. The same as Step 2 in the off-line phase.

3. Converting the received information as the input format of SVM, and SVM will use the model trained in the off-line phase to infer the location of the wireless client according to these information.

The other related details of the experiment will be described in the next section. 

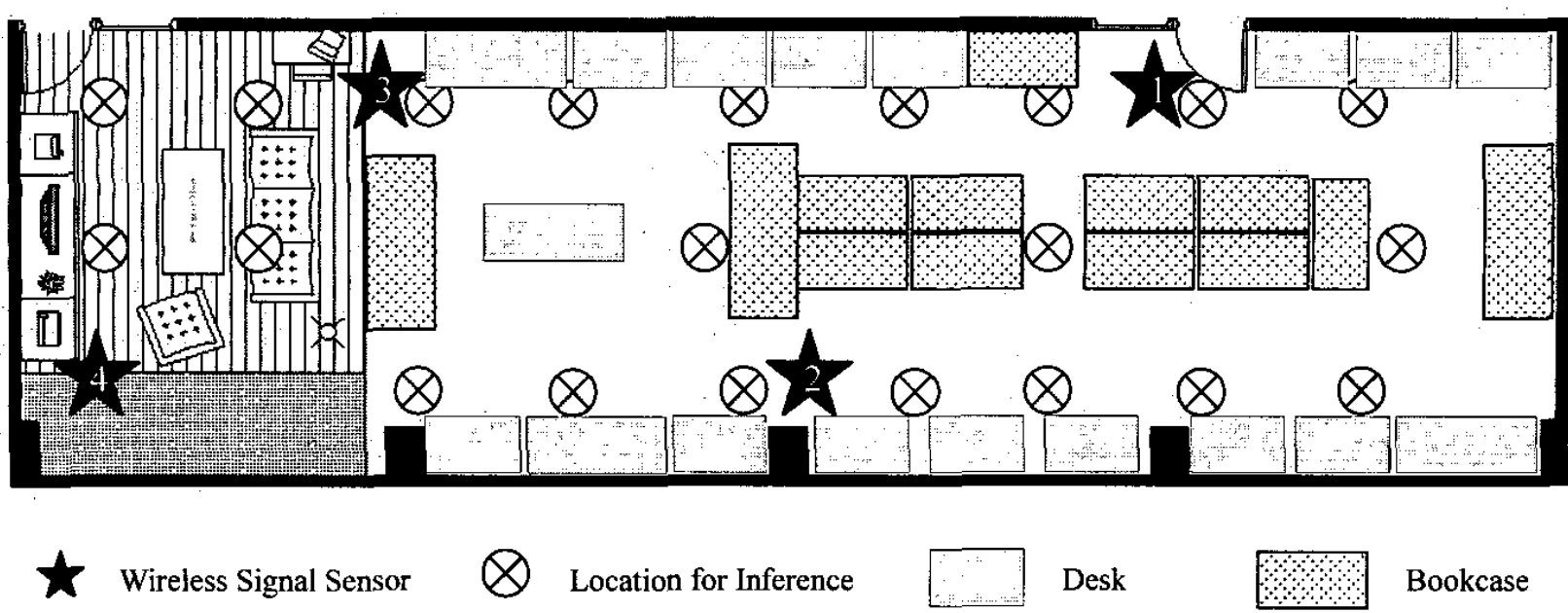

Figure 3. Configuration of the Intelligent Robot Laboratary

\section{Experiment}

All the experiments are set up at the Intelligent Robot Laboratory located in the Department of Computer Science and Information Engineering, National Taiwan University. The current e-Home experimental setup contains a home automation system controlling the resources of the whole laboratory. Three wireless signal sensors are used to set up the location determination system, and these wireless signal sensors are all located at the ceiling of the laboratory. Figure 3 shows the configuration of our experiment, and the locations we would like to determine are also marked. Each location is about 2 meters apart, and some locations are ignored because occupied by the desks or the bookcases.

According to previous research [9] - [12], at least three different information sources are needed to achieve better performance, and the system performance will be saturated when the number of sensors is greater than three. However, the fluctuation of the wireless signal is a very serious problem, so we use a fourth sensor to provide additional information to enhance the reliability. The performance of three sensors and four sensors are shown in the section 4 .

The sensor will detect multiple signal records from the same wireless client every second and then send them to the location inference server. When inferring the location of the wireless client, using the information retrieved at the same time instant is then very important. However, the network traffic may cause the delay of the immediate signal information, the record for location inference may also suffer from missing data, and hence the system will make the wrong determination. As a result, although the system will infer the location of each wireless client every second, the information used by SVM for inference purpose is retrieved and averaged over the latest two seconds to avoid the situation of missing data.

Figure 4 shows the signal strength fluctuation situation of one wireless client in the experiment tested. The unstable signal strength makes the determination of the wireless signal very difficult because the wireless signal strength of the personal digital device almost changes every second, and the changing range is very significant. As a result, the location inferred by the system may be incorrect if the changing range of the wireless signal is too significant.

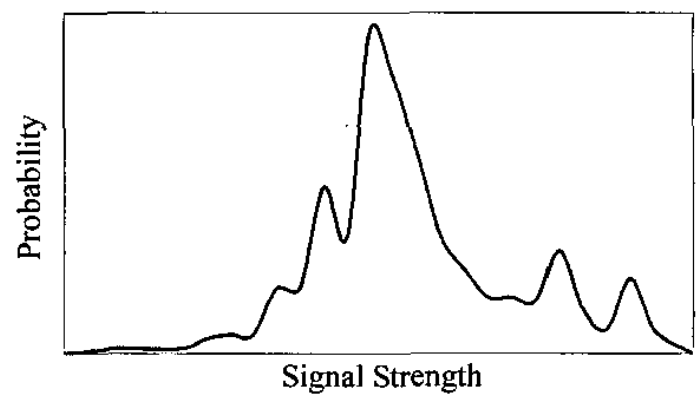

Figure 4. Signal Strength Fluctuation

To solve this problem, a simple mechanism is proposed to filter out the pulsing of the signal strength. The first time the location is determined different, the system will tend to assume that this situation is a false-positive. The system will not change the location in the database, but the location newly determined will be recorded as the potential location. If the location determined by the system at the next time period is the same as the potential one determined just now, the system will change the location in the database as the potential one. On the other hand, if the location determined at the next time period is different from the potential one, the system will not change the client location in the database. However, the 
potential location will be set as the newly determined one. Figure 5 shows the concept of this mechanism.

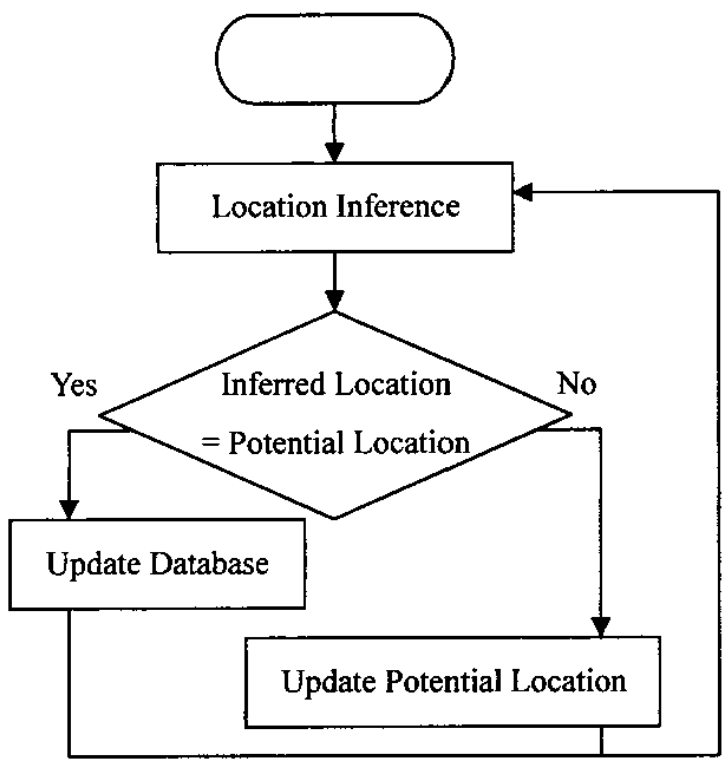

Figure 5. Filtering Mechanism

\section{Performance Evaluation}

When building the model for SVM, 300 records are sampled at each location marked in Figure 3. Figure 6 shows the performance of the cross validation of the prototype location determination system. The point marked as $(x, y)$ at the curve means that the probability to select a inference result with the error distance less than $x$ meters among all the inference results is $y$. The four curves correspond to the inference results using three/four sensors, and before/after applying the filtering mechanism, respectively.

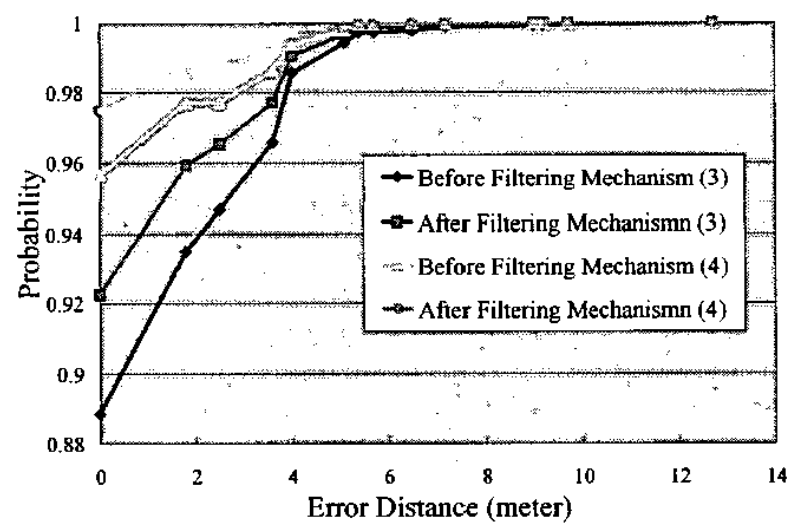

Figure 6. Cross Validation of the System Performance

Figure 7 shows the performance when the system infers the testing data. 50 records are sampled at each location to form the set of testing data. Although the system performance goes down when inferring testing data, the error distance in most instances is less than 2 meters. And this performance is still acceptable for the applications of e-Home when determining which system resource should be provided to the user because this range is already close to the usual activity area of people. Nevertheless, to achieve high performance when inferring testing data is still the goal for us to investigate, and we are working on it by several kinds of way. The related discussions are in the last section.

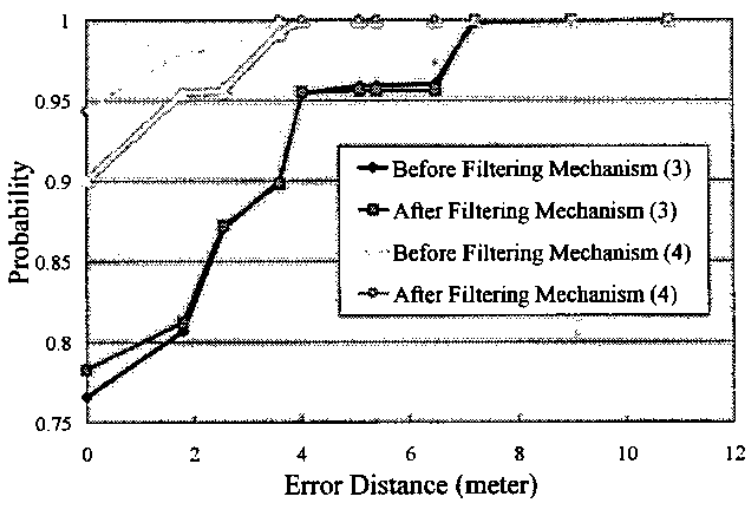

Figure 7. System Performance for the Testing Data

\section{Applications}

In this paper, three applications are considered when the location determination system cooperates with the home automation system. All these three applications have been implemented in our laboratory.

\subsection{Location Aware Service}

When one user would like to enter the laboratory but is locked out, he can just turn on his personal digital device outside the laboratory door, and use the device to connect the wireless network in the laboratory. Once the location determination system detects the signal of the personal device, it can tell whether the device belongs to the member of the laboratory, infer that the location of the device is outside the door, and inform the home automation system about this situation. Then the home automation system will automatically unlock the door for this user. The system will also lock the door and turn on the door lamp after the user enters the laboratory because the system will know that the user has been in the laboratory according to the location of the device.

\subsection{Personalized Service}

In the previous scenario, when the home automation system uses the resources neighbored the personal digital device to display the information to the user, it can provide personal 
related information (like the personal life for this user) since the user has been identified through her/his personal digital device. The system can also provide the user entertainment according to the preference of this user, such as turning on the TV and switching to his favorite channel automatically.

\subsection{Service Migration}

One user may roam around the living environment, and it will be wonderful if the user can access and enjoy the system services everywhere. The service migration is an important function in our home automation system [15]. With the information provided by the location determination system, the home automation system can know the location of the user and the system resource neighboring the user, and the services originally provided at the user's original location can be moved the living space neighboring the user, and provided by the neighbored system resources.

\section{Conclusion and Discussion}

In this paper, we presented a wireless network location determination system based on SVM, and this system has been implemented in our laboratory. Through cooperating with the home automation system, the e-Home framework can provide new services as we mentioned.

The limitation of the location determination system is that the inference model has to be built in advance. In other words, if there is some changes in the environment, the model may have to be rebuilt. Since the home environment may change everyday, it is an important issue to build an adaptive location determination system, and we are working on it right now.

The high fluctuation of the signal strength is the nature of the wireless network, and this is also a critical problem. Although we have proposed a simple method to filter out the pulsing of the signal strength, there is some other way to solve this problem [13]. There are also some other ways to infer the location [7][10-12], and integrating these solutions into current system may obtain better performance.

\section{Acknowledgement}

The authors would like to acknowledge the help from Hsin-Mu Tsai and Hung-Te Lin, who are students of the department of CSIE, National Taiwan University.

\section{References}

[1] Li-Chen Fu; Teng-Jei Shih; "Holonic supervisory control and data acquisition kernel for 21 st century intelligent building system", IEEE International Conference on Robotics and Automation, 2000.

[2] Wen-Ya Chung; Li-Chen Fu; Shih-Shinh Huang; "A flexible, hierarchical and distributed control kernel architecture for rapid resource integration of intelligent building system", IEEE International Conference on Robotics and Automation, 2001.

[3] Neng-Shiang Liang; Li-Chen Fu; Chao-Lin Wu, "An integrated, flexible, and Internet-based control architecture for home automation system in the Internet era", IEEE International Conference on Robotics and Automation, 2002.

[4] Want, R.; Hopper, A.; "Active badges and personal interactive computing objects", Consumer Electronics, IEEE Transactions on , Volume: 38 Issue: 1 , Feb. 1992, Page(s): $10-20$

[5] Krumm, J.; Harris, S.; Meyers, B.; Brumitt, B.; Hale, M.; Shafer, S.; "Multi-camera multi-person tracking for EasyLiving", Proceedings. Third IEEE International Workshop on Visual Surveillance, 2000

[6] Enge, P.; Misra, P.; "Special Issue on Global Positioning System", Proceedings of the IEEE, Volume: 87 Issue: 1, Jan. 1999

[7] Paul Castro, Patrick Chiu, Ted Kremenek, and Richard Muntz, "A Probabilistic Location Service for Wireless Network Environments," In Proceedings of Ubicomp 2001, pp. 18--24. Springer Verlag, September 2001.

[8] Paramvir Bahl and Venkata N. Padmanabhan, "RADAR: An In-Building RF-based User Location and Tracking System," IEEE Infocom 2000, volume 2, pages 775-784, March 2000

[9] P. Bahl, V. N. Padmanabhan, and A. Balachandran, "Enhancements to the RADAR User Location and Tracking System," Microsoft Research Technical Report: MSR-TR-00-12 (February 2000)

[10] Prasithsangaree, P.; Krishnamurthy, P.; Chrysanthis, P.; "On indoor position location with wireless LANs", Personal, Indoor and Mobile Radio Communications, 2002. The 13th IEEE International Symposium on , Volume: 2 , 15-18 Sept. 2002, Page(s): $720-724$ vol.2

[11] M. Youssef, A. Agrawala, A. U. Shankar, and S. H. Noh, "A Probabilistic Clustering-Based Indoor Location Determination System," University of Maryland, College Park, Tech. Rep. UMIACSTR, 2002-30 and CS-TR 4350, March 2002.

[12] Youssef, M.A.; Agrawala, A.; Shankar, A.U.; "WLAN location determination via clustering and probability distributions", Pervasive Computing and Communications, 2003. Proceedings of the First IEEE International Conference on , 23-26 March 2003, Page(s): 143 $-150$

[13] Youssef, M.; Agrawala, A.; "Small-scale compensation for WLAN location determination systems", Wireless Communications and Networking, 2003. Volume: 3, 16-20 March 2003, Page(s): 1974 $-1978$

[14] K. Cheverst, etal. "Experiences of Developing and Deploying a Context-Aware Tourist Guide: The GUIDE Project", Proceedings of the sixth annual international conference on Mobile computing and networking, August 2000

[15] Wei-Cheng Wang, Chao-Lin Wu, Li-Chen Fu, "Adaptive Home Automation System through Dynamically Changing the System's Architecture"

[16] Chih-Chung Chang and Chih-Jen Lin, LIBSVM : a library for support vector machines, 2001. Software available at http:/www.csie.nturedu.tw/-cjlin/libsvm 